\title{
Optical Absorption and Raman Scattering Studies of Few-Layer Epitaxial Graphene Grown on 4H-SiC Substrates
}

\author{
K. Grodecki ${ }^{a, b}$,A. Drabińska ${ }^{a}$, R. BożeK ${ }^{a}$, A. WysmoŁek ${ }^{a}$, K.P. Korona ${ }^{a}$, \\ W. Strupiński ${ }^{b}$, J. BorysiuK $^{a}$, R. StęPniEWski ${ }^{a}$ AND J.M. BARANOWski ${ }^{a, b}$ \\ ${ }^{a}$ Institute of Experimental Physics, Faculty of Physics, University of Warsaw, Hoża 69, 00-681 Warsaw, Poland \\ ${ }^{b}$ Institute of Electronic Materials Technology, Wólczyńska 133, 01-919 Warsaw, Poland
}

\begin{abstract}
Optical absorption and Raman scattering studies of few-layer epitaxial graphene obtained by high temperature annealing of carbon terminated face of $4 \mathrm{H}-\mathrm{SiC}(000-1)$ on-axis substrates are presented. Changing the pressure and annealing time, different stages of the graphene formation were achieved. Optical absorption measurements enabled us to establish average number of graphene layers covering the $\mathrm{SiC}$ substrate. Raman scattering experiments showed that integrated intensity of the characteristic $2 \mathrm{D}$ peak positively correlated with the number of graphene layers deposited on the $\mathrm{SiC}$ substrate. The spectral width of the $2 \mathrm{D}$ peak was found to decrease with the number of the deposited graphene layers.
\end{abstract}

PACS numbers: 78.66.Tr, 78.40.Ri, 78.30.Na, 63.20.dd, 63.20.kd

\section{Introduction}

Production of graphene by annealing of silicon carbide $(\mathrm{SiC})$ substrates has recently attracted a lot of interest. Opposite to the mechanical exfoliation [1], the annealing of $\mathrm{SiC}$ substrates [2] is more compatible with nowadays industrial processing techniques. However, reaching large area coverage with controlled number of deposited graphene layers still is a big challenge. The growth of graphene structures depends on many parameters including pressure, temperature and annealing time during graphitization process, as well as depends on polytype, polarity and preparation of the $\mathrm{SiC}$ substrates. On the contrary to the Raman spectroscopy, which has been proved to be very efficient and useful method to study graphene structures and other graphite-like allotropes [3], optical absorption is quite novel method used for graphene characterization. Despite being only one-atom thick, single graphene layer was found to absorb a significant fraction (about 2.3\%) of incident white light [4]. This makes the optical absorption an effective tool for evaluation of single- and multi-layer graphene formation. In this communication comparison of the optical absorption and Raman scattering studies of few layer epitaxial graphene, obtained by high temperature annealing of carbon terminated face of $4 \mathrm{H}-\mathrm{SiC}(000-1)$ on-axis substrates, are presented.

\section{Experimental details}

The graphene layers were grown on carbon face $4 \mathrm{H}-\mathrm{SiC}$ substrates using Epigres V508 hot wall chemical vapour deposition (CVD) reactor with a $\mathrm{SiC}$ and $\mathrm{TiC}$ coated graphite susceptors, heated by RF generator. Before the growth of graphene the $\mathrm{SiC}$ substrates were etched at $1600^{\circ} \mathrm{C}$ in atmosphere of hydrogen and propane. After etching, process of graphitisation (annealing) was done at $1600{ }^{\circ} \mathrm{C}$ in argon atmosphere. In this work two series of samples have been studied. For the first one, the same annealing (graphitization) time (10 min) have been used, whereas different argon pressure between 5 mbar to 800 mbar in the reactor have been kept during the growth process. The second series of samples have been obtained keeping the same pressure of argon of $100 \mathrm{mbar}$, however the graphitization time was varied from $10 \mathrm{~min}$ to $40 \mathrm{~min}$. Atomic force microscopy (AFM) images were recorded in air at ambient temperature. Micro-Raman scattering experiments were performed at room temperature, in backscattering geometry, using $532 \mathrm{~nm}$ line from Nd-YAG laser as a source of continuous wave excitation. Laser spot size on the sample surface was about $2 \mu \mathrm{m}$. The excitation power was below $50 \mathrm{~mW}$ to avoid surface heating. For all investigated samples optical absorption measurements have been performed in the spectral range between 500 and $1100 \mathrm{~nm}$. In order to establish the net absorption of the graphene layers, the measured signals have been corrected for the absorption of the $\mathrm{SiC}$ substrate. Cross-sectional transmission electron microscopy (TEM) specimens were prepared by a standard method of mechanical pre-thinning followed by Ar ion milling. The contrast and high resolution TEM observations were performed on a JEOL JEM 3010 microscope operating at $300 \mathrm{kV}$.

\section{Experimental results and discussion}

The AFM image of two samples grown at 100 mbar for $10 \mathrm{~min}$ and at $5 \mathrm{mbar}$ for $10 \mathrm{~min}$, respectively, are shown 
in Fig. 1. It is observed that the size of graphene flakes varies from $1 \mu \mathrm{m}$ up to $10 \mu \mathrm{m}$ depending on the graphitisation time and argon pressure. The biggest flake size is obtained for the lowest pressures and longest annealing times.

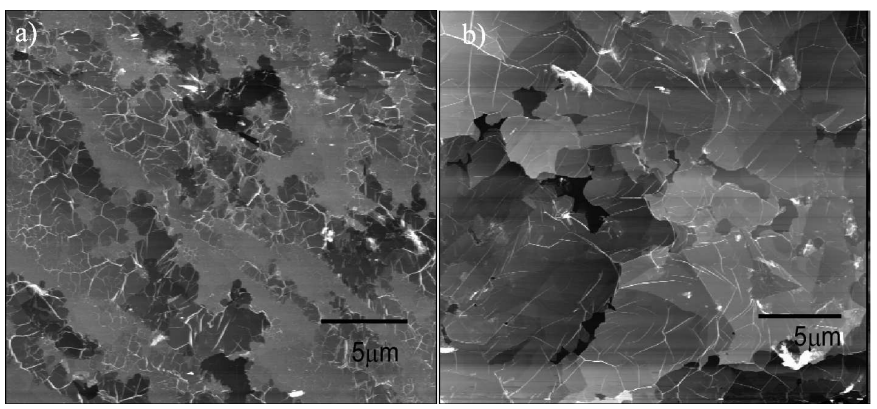

Fig. 1. AFM image of the sample grown in (a) 100 mbar and $10 \mathrm{~min}$ and sample grown in (b) 5 mbar and $10 \mathrm{~min}$. White lines seen in images correspond to "puckers" [15].

Transmission $(T)$ spectra were measured for the wavelength range 600-1000 nm. Typical dimensionless absorption $(A)$ spectrum (calculated as $A:=1-T$ ) obtained for the sample grown at $100 \mathrm{mbar}$ for $40 \mathrm{~min}$ is presented in Fig. 2. It is observed that for whole spectral range the absorption of graphene is nearly flat, and with the mean value of about 0.19 for the range 600-1000 nm. This is in agreement with theoretical calculations which show that the optical absorption (transmission) of the single graphene layer in visible spectral range should be wavelength independent [5].

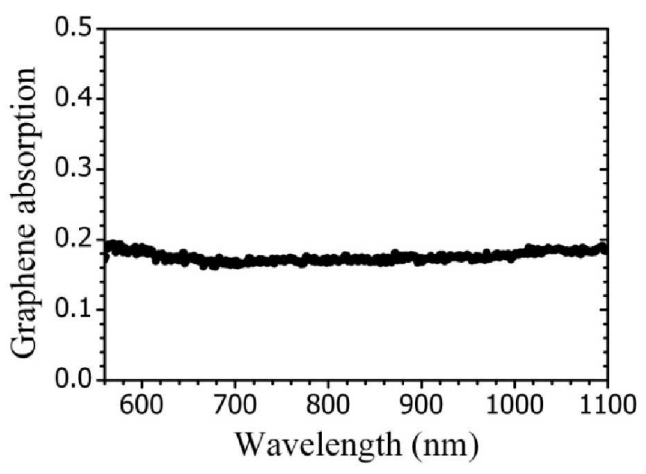

Fig. 2. Optical absorption spectrum of the sample grown in 100 mbar for $40 \mathrm{~min}$.

In order to calculate the transmittance of a graphene layer on $\mathrm{SiC}$ substrate one has to consider interface effects between air and $\mathrm{SiC}$ with boundary conditions for the electromagnetic field that are modified by the presence of the conducting graphene layer. Normalization to the bare $\mathrm{SiC}$ substrate makes it independent of substrate thickness. It was already shown that the transmission through $N$ graphene layers deposited on $\mathrm{SiC}$ substrate normalized to the transmission through bare $\mathrm{SiC}$ can be expressed as [6]:

$$
T=\left(1+\frac{N \sigma_{0}}{1+n_{\mathrm{SiC}}} \sqrt{\frac{\mu_{0}}{\varepsilon_{0}}}\right)^{-2},
$$

where $\sigma_{0}=e^{2} /(4 \hbar)$ is interband conductivity and $n_{\mathrm{SiC}}=2.55$ stands for the refractive index of $\mathrm{SiC}$. In the limit of $N \sigma_{0} \sqrt{\mu_{0} / \varepsilon_{0}} \ll 1+n_{\mathrm{SiC}}$ (which corresponds to the condition $N \ll 150$ ), the normalized absorption can be expressed as

$$
A=1-T=\frac{2 N \sigma_{0}}{1+n_{\mathrm{SiC}}} \sqrt{\frac{\mu_{0}}{\varepsilon_{0}}}=0.0129 N .
$$

According to Eq. (2) the mean absorption value of 0.19 measured for the sample, whose spectrum is presented in Fig. 2, corresponds to 15 layers of graphene.

This result is in very good agreement with TEM results obtained on this sample presented in Fig. 3.

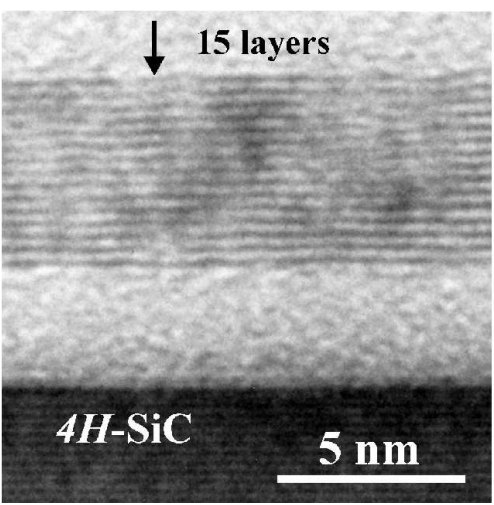

Fig. 3. TEM image of sample grown in 100 mbar for $40 \mathrm{~min}$.

It was found that samples with largest graphene flake sizes consist of the largest number of graphene layers. This observation is consistent with the results of the Raman scattering experiments. Since in the case of epitaxial graphene the characteristic excitations [3] (except 2D band) overlap with strong background related to the second order of $\mathrm{SiC}$ spectrum [7-14], we focused our analysis on discussion of the properties of the so-called $2 \mathrm{D}$ band (see Fig. 4).

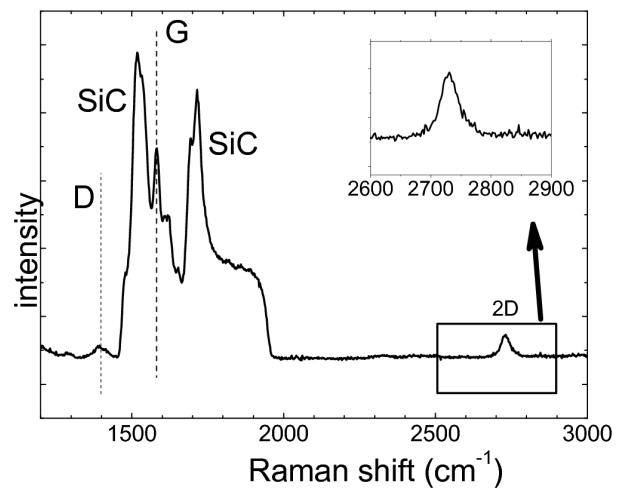

Fig. 4. Raman spectrum of epitaxial graphene. In the inset the enlarged part of the spectrum is shown. 


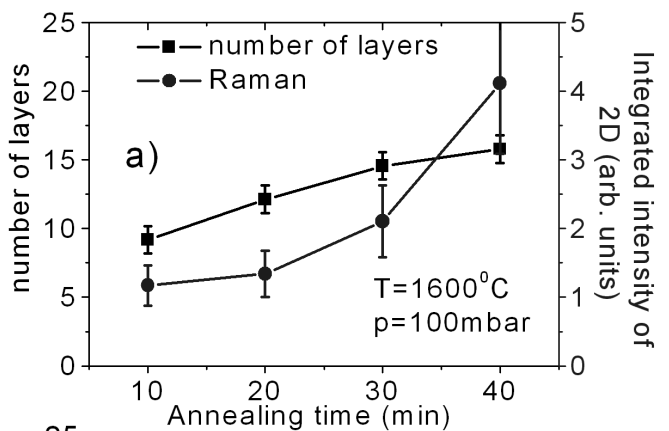

with annealing time and decrease with argon pressure in the reactor.

\section{Acknowledgments}

The work was partially supported by Polish Ministry of Science and Higher Education, Grants PBZ-MEiN-6/2/2006 and DWM/N179/PICS-FR/2008 and EU Project No. MTKD-CT-2005-029671.

\section{References}

[1] K.S. Novoselov, A.K. Geim, S.V. Morozov, D. Jiang, Y. Zhang, S.V. Dubonos, I.V. Grigorieva, A.A. Firsov, Science 306, 666 (2004).

[2] C. Berger, Z. Song, T. Li, X. Li, A.Y. Ogbazghi, R. Feng, Z. Dai, A.N. Marchenkov, E.H. Conrad, P.N. First, W.A. Heer, J. Phys. Chem. B 108, 19912 (2004).

[3] C. Faugeras, A. Nerrière, M. Potemski, A. Mahmood, E. Dujardin, C. Berger, W.A. de Heer, Appl. Phys. Lett. 92, 011914 (2008).

[4] R.R. Nair, P. Blake, A.N. Grigorenko, K.S. Novoselov, T.J. Booth, T. Stauber, N.M.R. Peres, A.K. Geim, Science 320, 1308 (2008).

Fig. 5. Integrated intensity of $2 \mathrm{D}$ band and number of layers as a function of (a) annealing time, (b) pressure.

Figure 5 presents comparison of integrated intensity of $2 \mathrm{D}$ peak in the Raman spectrum and number of layers obtained from optical absorption measurements depending on annealing time and argon pressure in the reactor. Transmission measurements shows that number of layers increases with increasing graphitization time (Fig. 5a) and decreases with increasing pressure in the reactor (Fig. 5b). The same behavior with pressure and graphitization time is observed for integrated intensity of $2 \mathrm{D}$ band. It means that integrated intensity of $2 \mathrm{D}$ band and thickness of graphene structures are closely correlated. This observation can be very useful for characterisation of graphene structures grown on $\mathrm{SiC}$ substrates.

\section{Conclusions}

In this work we presented two series of samples with different growth conditions. The first with different argon pressure in the reactor and the second with different annealing time. We have found direct correlation between optical absorption measurement and Raman scattering for various samples which differ in graphitisation time and pressure of argon in growth reactor. Both numbers of layers obtained from absorption measurement and integrated intensity of $2 \mathrm{D}$ peak in Raman spectrum increase

[5] T. Stauber, N.M.R. Peres, A.K. Geim, Phys. Rev. B 78, 085432 (2008).

[6] J.M. Dawlaty, S. Shivaraman, J. Strait, P. George, M. Chandrashekhar, F. Rana, M.G. Spencer, D. Veksler, Y. Chen, Appl. Phys. Lett. 93, 131905 (2008).

[7] C. Berger, Z.M. Song, X.B. Li, X.S. Wu, N. Brown, C. Naud, D. Mayo, T.B. Li, J. Hass, A.N. Marchenkov, E.H. Conrad, P.N. First, W.A. de Heer, Science 312, 1191 (2006).

[8] A.K. Geim, K.S. Novoselov, Nature Mater. 6, 183 (2007).

[9] J. Borysiuk, R. Bożek, W. Strupiński, A. Wysmołek, K. Grodecki, R. Stępniewski, J.M. Baranowski, J. Appl. Phys. 105, 023503 (2009).

[10] Z.H. Ni, W. Chen, X.F. Fan, J.L. Kuo, T. Yu, A.T.S. Wee, Z.X. Shen, Phys. Rev. B 77, 115416 (2008).

[11] J. Röhrl, M. Hundhausen, K.V. Emtsev, Th. Seyller, R. Graupner, L. Ley, Appl. Phys. Lett. 92, 201918 (2008).

[12] N. Ferralis, R. Maboudian, C. Carraro, Phys. Rev. Lett. 101, 156801 (2008).

[13] A.C. Ferrari, Solid State Commun. 143, 47 (2007).

[14] A.C. Ferrari, J.C. Meyer, V. Scardaci, C. Casiraghi, M. Lazzeri, F. Mauri, S. Piscanec, D. Jiang, K.S. Novoselov, S. Roth, A.K. Geim, Phys. Rev. Lett. 97, 187401 (2006).

[15] J. Hass, W.A. de Heer, E.H. Conrad, J. Phys., Condens. Matter 20, 323202 (2008). 